\title{
Die Aktivitäten der POA im Jahr 2013
}

Die Pneumologisch-onkologische Arbeitsgemeinschaft (POA) in der Deutschen Krebsgesellschaft e.V. (DKG) umfasst knapp 100 Mitglieder und ist interdisziplinär zusammengesetzt, insbesondere aus den an der Therapie des Lungenkarzinoms beteiligten Disziplinen. Unter den Mitgliedern am breitesten vertreten ist die Fachdisziplin der Pneumologie. Die POA sieht ihre Ziele in der Sicherung der Qualität der Behandlung von Patienten auf dem Gebiet der Thoraxonkologie. Zudem vertritt sie die onkologische Pneumologie bei wissenschaftlichen Tagungen, wirkt an der Entwicklung von Leitlinien mit, an der Weiterentwicklung der klinischen Forschung wie auch Grundlagenwissenschaft auf dem Gebiet und berät fachlich Gremien und Institutionen zu Inhalten der Thoraxonkologie. So hat die POA im Jahr 2013 gemeinsam mit der Deutschen Gesellschaft für Pathologie ein „Positionspapier zur Verbesserung der onkologischen Versorgung krebskranker Patienten mittels personalisierter Tumormedizin“ auf den Weg gebracht (u.a. als Publikation im DKG-Forum und damit einen Diskussionsprozess in der DKG angestoßen. Dieser wird nun mit dem Ziel, die Notwendigkeiten der molekularen Diagnostik in der Onkologie generell im Kontext der Gesundheitspolitik zu adressieren, breit geführt.

Den Deutschen Krebskongress (DKK) im Februar 2014 hatte die POA im Hinblick auf die Themenfelder der Thoraxonkologie maßgeblich konzeptionell gestaltet, wie auch durch inhaltliche Expertise in der konkreten Umsetzung zum hohen $\mathrm{Ni}$ veau auf diesem Gebiet beigetragen. Im „Trail“ Thoraxonkologie des DKK hat die POA 9 von 12 Symposien organisiert und zu weiteren 2 Symposien beigetragen.

\section{Interdisziplinäres Netzwerk Thoraxonkologie (INT)}

Zur Förderung der Interaktion zwischen den an thoraxonkologischen Erkrankungen in Diagnostik und Therapie beteiligten Disziplinen verfolgt die POA seit 2010 die „INT-Aktivität“. Diese Plattform bietet ein Forum für interessierte Kollegen, die auf dem Feld der Thoraxonkologie aktiv sind. Dazu findet 2-mal jährlich ein halbtägiges Meeting statt. Die Schwerpunkte werden jeweils von den Teilnehmern des aktuellen
Meetings für das nächste stattfindende Forum festgelegt. Auf Basis der interdisziplinären Interaktion soll die INT-Plattform insbesondere den „klinischen Mittelbau“ ansprechen, Gedanken und Ideen aufgreifen und Impulse konzeptionell strukturieren, um so das Feld in der Thoraxonkologie in der DKG realitätsnah weiter zu bewegen.Themen, die in den letzten beiden Jahren aufgegriffen wurden, waren u.a.: Versorgungsstrukturgesetz und dessen Bedeutung für die Thoraxonkologie, Krebsinformationsdienst in der Thoraxonkologie - Angebot für Bevölkerung und Fachkreise, Shift stationär/ambulant im Kontext des $\S 116$ b, Ernährungskonzepte beim Lungenkrebskarzinom, Stellenwert und Konzept der frühen palliativen Führung sowie molekulare Diversifikation beim Lungenkarzinom.

\section{„Februar-Symposium“ Thoraxonkologie}

$\checkmark$

Seit 2009 veranstaltet die POA jährlich ein Symposium, das die aktuellen Themen der Thoraxonkologie aufgreift. Mit Übernahme der Sprecherfunktion von Prof. Michael Thomas, Heidelberg, und Dr. Karl-Matthias Deppermann, Erfurt, wird die Veranstaltung seit 2011 interdisziplinär unter Einbindung der auf dem Feld der Thoraxonkologie aktiven Arbeitsgemeinschaften in der DKG wie auch der Deutschen Gesellschaft für Pneumologie (DGP) gestaltet. Mittlerweile hat dieses Meeting in Fachkreisen eine gute Reputation und wird breiter wahrgenommen. So nahmen an dem diesjährigen „Februar-Smposium“ mehr als 160 Personen teil. Die Themenwahl, insbesondere die Diskussionsatmosphäre und Offenheit des Austausches werden als aktuell und kompetent mit niedriger Diskussionsschwelle eingeschätzt. Damit hat die POA mit diesem Format das Ziel eines unkomplizierten, lebendigen und kompetenten Austauschs auf hohem Niveau erreicht. Dies soll künftig- hin weiter gepflegt und an der ein oder anderen Stelle noch verbessert und angepasst werden.

Prof. Michael Thomas, Heidelberg

Kohlenmonoxid

\section{Polymer-Vlies macht Gas kontrollierbar}

Kohlenmonoxid (CO) ist zwar ein giftiges Gas und verhindert - einmal eingeatmet den Sauerstofftransport im Blut, doch könnte es dank eines neuen Polymer-Vlieses in immer mehr Bereichen der modernen Medizin zum Einsatz kommen. Denn CO ist nicht nur schädlich für den Organismus, es hat auch nützliche Eigenschaften, wie eine entzündungshemmende Wirkung. Zudem schützt das Gas die Organe bei Organtransplantationen vor Zellschäden. „Doch die sich daraus ergebenden vielversprechenden Anwendungsmöglichkeiten sind bisher in der Praxis nicht umsetzbar. Voraussetzung für einen solchen Einsatz von CO wäre es, das Gas kontrolliert und ausschließlich am gewünschten Ort zu applizieren", meint Alexander Schiller von der Universität Jena . Der Chemiker hat zu diesem Zweck nun ein lichtsensibles Polymer-Vlies vorgestellt. Dieses setzt CO kontrolliert frei und ist damit prinzipiell als Material für biomedizinische Anwendungen geeignet. Details der Studie wurden im Februar im Journal of Materials Chemistry B veröffentlicht. Bei der Neuentwicklung handelt es sich um eine Metall-Carbonyl-Verbindung, die zusammen mit einem Polymer zu einer Faser von etwa einem Mikrometer Durchmesser gesponnen wird, aus der ein dichtes zweidimensionales Vlies entsteht. Der integrierte „Lichtschalter" ist wichtig: Wird das Polymer mit violettem oder blauem Licht bestrahlt, setzt es CO-Gas frei. Wegen der präzisen Steuerung der Gasabgabe ließe sich das inzwischen patentierte System auch zur Eichung von Gassensoren nutzen.

pte 